\title{
Notes on the Lie symmetry exact explicit solutions for nonlinear Burgers' equation
}

\author{
Bohua $\mathrm{SUN}^{1}$ \\ ${ }^{1}$ School of Civil Engineering 8 Institute of Mechanics and Technology, \\ Xi'an University of Architecture and Technology, Xi'an 710055, China \\ http://imt.xauat.edu.cn \\ sunbohua@xauat.edu.cn \\ (Dated: August 12, 2019)
}

In light of Liu's original works, this paper revisits the solution of general Burgers's nonlinear equation. We obtain two exact and explicit solutions for group $G_{4}$ and $G_{6}$, and a most general solution as well. As applications, a numerical example is carried out.

Keywords: Lie group, Burgers equation, exact solution, general solution, elementary function

\section{Contents}

\section{Introduction}

II. Lie infinitesimal generator, Lie algebra and Lie group of $u_{t}=a\left(u_{x}\right)^{2}+b u_{x x}$

III. Exact general solutions of $u_{t}=a\left(u_{x}\right)^{2}+b u_{x x}$

IV. Exact similarity solutions of $u_{t}=a\left(u_{x}\right)^{2}+b u_{x x}$

V. Numerical calculations of $u_{t}=\left(u_{x}\right)^{2}+u_{x x}$ and discussions

VI. Acknowledgement

References

\section{INTRODUCTION}

Most of the problems in science and engineering are nonlinear in nature, and their corresponding governing equations are nonlinear equations. Solving them is an important task. It is well known that nonlinear partial differential equations have infinitely number solutions, and most of them are variable coefficient equations. Therefore, it is very difficult to obtain their accurate solutions. Although there are few methods for solving nonlinear partial differential equations, but for variable-coefficient nonlinear partial differential equations, the current research methods only stay in numerical solution or approximate solution. Around 1875, Norwegian mathematician Sophus Lie [1-3] proposed a method of continuous transform group, which can simplify the original equation to obtain an exact solution through Lie group or symmetry group transformation. This method does not require special transformation techniques. It is a systematic method that is applicable not only to ordinary differential equations but also to partial differential equations. It is currently the most powerful analytical tool. Cantwell [4] even initiates: students in science and engineering should learn both dimension analysis and Lie group (symmetric analysis), the former is for establishing a physical model (equation), and the latter is for solving or analyzing the structure of the solution [27].

In mathematics, Lie group is a group and also a differential manifold with the property of maintaining a smooth structure under group operation. There is a relatively rich literature on Lie group analysis. Lie began to create a single-parameter continuous group theory (later known as the Lie group) around 1875, and completed the three-step continuation of the famous monographs about Lie group [1-3]. After publication of Lie's three volume monographs, the first textbook on the Lie group was completed in 1897 by American scholar J.M. Page [5], who was one of the first students to attend Lie's lecture on the Lie group. American scholar A. Cohen published the second textbook in 1911 [6]. Ince, a scholar at the American University of Egypt in 1926, introduced the Lie group symmetry method in his ordinary differential equation book [7]. After a long period of silence, in 1960 American scholar Birkhoff published a monograph on the study of fluid mechanics with the Lie group. Since the high-level study presented by Birkhoff, the Lie group symmetry method has begun to attract more attention and enter a high-speed application. development stage [8]. In the application development of the Lie group symmetry method, it must especially mention that the Lie 
group analysis monograph [9] of the differential equation of the former Soviet scholar Ovsiannikov, who leaded Soviet school have promoted the development of Lie group application and computer computing. Ovsiannikov's monograph was translated into English by Bluman in 1967. Hansen's book in 1964 discusses fluid mechanics and heat transfer problems. In 1965, Ames' monograph studied nonlinear problems in engineering [10]. Ibragimov and Bluman later made an influential contribution to the education and application of the Lie group [11-21, 24-27].

Liu, et al. [23] applied the Lie group to study the general nonlinear Burgers equation [22]:

$$
u_{t}=a\left(u_{x}\right)^{2}+b u_{x x} .
$$

where $u=u(x, t)$ is a real function, and parameters $a, b \in R$ with $a b \neq 0$, and $u_{t}=\frac{\partial}{\partial t}, u_{x}=\frac{\partial}{\partial x}$ and $u_{x x}=\frac{\partial^{2}}{\partial x^{2}}$.

For the general nonlinear Burgers equation, Liu, et al. [23] obtained six one-parameter Lie group $G_{i}, i=1 \ldots 6$, four exact explicit solutions corresponding the group $G_{1}, G_{2}, G_{3}$ and $G_{5}$. Unfortunately, Liu, et al. [23] could not find the exact and explicit solutions for both group $G_{4}$ and $G_{6}$. For group $G_{4}$, Liu, et al. [23] left an unfinished integration:

$$
f(\xi)=\int \frac{e^{-\frac{\xi^{2}}{4 b}}}{\int e^{-\frac{\xi^{2}}{4 b^{2}}}+p_{1}} d \xi+p_{2} .
$$

For the group $G_{6}$, Liu, et al. [23] derived a nonlinear ordinary differential equation:

$$
4 a b \theta^{2} f^{\prime \prime}+4 a^{2} \theta^{2} f^{\prime 2}-4 a b \theta f^{\prime}+3 b^{2}=0 .
$$

Liu, et al. [23] states that "we cannot obtain the exact and explicit solutions for Eq.(3) by using the elementary functions.". To solve the Eq.(3), they cleverly proposed a generalized power series solution: $f(\xi)=\alpha \log |\xi|+\beta+$ $\sum_{n=0}^{\infty} f_{n} \xi^{n+1}$, and took a quite effect to determine all coefficients.

General speaking, the results of Liu, et al. [23] are nice and will be more beautiful if the solutions for both group $G_{4}$ and $G_{6}$ can be obtained and expressed in elementary functions. We will make up this gap in this paper.

The paper is organized as follows: for self-contained purpose, following the introduction we summary some results of Liu, et al. [23], including Lie symmetric infinitesimal generator, Lie algebra; and in Section 3, we presents a most general solution for $u_{t}=a\left(u_{x}\right)^{2}+b u_{x x}$; in Section 4 , two exact nd explicit solutions will be proposed. In Section 5 , as application, a numerical example has been carried out and results are plotted. Finally, the paper concludes with perspectives about the future development of research studies.

\section{LIE INFINITESIMAL GENERATOR, LIE ALGEBRA AND LIE GROUP OF $u_{t}=a\left(u_{x}\right)^{2}+b u_{x x}$}

First, do an infinitesimal transformation on $x, t, u$

$$
\begin{aligned}
x & \mapsto x+\epsilon \xi(x, t, u), \\
t & \mapsto t+\epsilon \tau(x, t, u), \\
u & \mapsto u+\epsilon \phi(x, t, u) .
\end{aligned}
$$

The corresponding infinitesimal generator $X$ can be expressed as

$$
X=\xi \frac{\partial}{\partial x}+\tau \frac{\partial}{\partial t}+\phi \frac{\partial}{\partial u}
$$

and the 2nd order prolongation $X^{(2)}$ is given

$$
X^{(2)}=X+\phi^{t} \frac{\partial}{\partial u_{t}}+\phi^{x} \frac{\partial}{\partial u_{x}}+\phi^{x x} \frac{\partial}{\partial u_{x x}},
$$

where

$$
\begin{aligned}
\phi^{t} & =D_{t} \phi-u_{x} D_{t} \xi-u_{t} D_{t} \tau \\
\phi^{x} & =D_{x} \phi-u_{x} D_{x} \xi-u_{t} D_{x} \tau \\
\phi^{x x} & =D_{x}^{2} \phi-u_{x} D_{x}^{2} \xi-u_{t} D_{t}^{2} \tau-2 u_{x x} D_{x} \xi-2 u_{x t} D_{x} \tau
\end{aligned}
$$


and $D_{x}, D_{t}$ are the total deferential respect to $x, t$, respectively

$$
\begin{aligned}
D_{x} & =\frac{\partial}{\partial x}+u_{x} \frac{\partial}{\partial u}+u_{x x} \frac{\partial}{\partial u_{x}}+u_{x t} \frac{\partial}{\partial u_{t}}, \\
D_{t} & =\frac{\partial}{\partial t}+u_{t} \frac{\partial}{\partial u}+u_{x t} \frac{\partial}{\partial u_{x}}+u_{t t} \frac{\partial}{\partial u_{t}} .
\end{aligned}
$$

The Lie group symmetry of the equation (1) is

$$
X^{(2)}\left(u_{t}-a u_{x}^{2}-b u_{x x}\right)=0, \quad \text { ie. } \quad \phi^{t}-2 a u_{x} \phi^{x}-b \phi^{x x}=0 .
$$

Substituting the relevant expression into the symmetry determining equation (14), paying attention to replacing $u_{t}$ with $a u_{x}^{2}+b u_{x x}$, and comparing the coefficient functions that can get infinitesimal generators (you can also use Maple obtained)

$$
\begin{aligned}
\xi & =4 a c_{1} x t+c_{2} x+2 a c_{4} t+c_{5} \\
\tau & =4 a c_{1} t^{2}+2 c_{2} t+c_{3}, \\
\phi & =a e^{-a u / b}-c_{1} x^{2}-c_{4} x-2 b_{1} t+c_{6} .
\end{aligned}
$$

Where $c_{i}(i=1,2, \cdots, 6)$ is an arbitrary constant. $\alpha=\alpha(x, t)$ is a function that satisfies the following conduction equation

$$
\alpha_{t}=b \alpha_{x x}
$$

So we have the problem of infinitesimal generators.

$$
X=\sum_{i=1}^{6} c_{i} X_{i}+V_{\alpha} .
$$

where the base vector are

$$
\begin{array}{r}
X_{1}=\frac{\partial}{\partial x}, \quad X_{2}=\frac{\partial}{\partial t}, \quad X_{2}=\frac{\partial}{\partial u} \\
X_{4}=x \frac{\partial}{\partial x}+2 t \frac{\partial}{\partial t}, \quad X_{5}=2 a t \frac{\partial}{\partial x}-x \frac{\partial}{\partial u} \\
X_{6}=4 a x t \frac{\partial}{\partial x}+4 a t^{2} \frac{\partial}{\partial t}-\left(x^{2}+2 b t\right) \frac{\partial}{\partial u} \\
X_{\alpha}=\alpha(x, t) e^{-a u / b} \frac{\partial}{\partial u} .
\end{array}
$$

Where $X_{\alpha}$ is an infinite dimensional subalgebra。

Now verify that $X_{i}$ satisfies the closure of Lie algebra, ie $X_{i}$, and $X_{j} \in \mathcal{L}$, then $\left[X_{i}, X_{j}\right] \in \mathcal{L}$. The vector field exchange sub- $\left[X_{i}, X_{j}\right]$ structure coefficient list 15.3 is as follows:

TABLE I: Burgers equation Lie algebra structure coefficient table

\begin{tabular}{cccccccc}
\hline$\left[X_{i}, X_{j}\right]$ & $X_{1}$ & $X_{2}$ & $X_{3}$ & $X_{4}$ & $X_{5}$ & $X_{6}$ & $X_{\alpha}$ \\
\hline$X_{1}$ & 0 & 0 & 0 & $-X_{1}$ & $X_{3}$ & $-2 X_{5}$ & $-X_{\alpha_{x}}$ \\
$X_{2}$ & 0 & 0 & 0 & $-2 X_{2}$ & $-2 a X_{1}$ & $2 b X_{3}-4 a X_{4}$ & $-X_{\alpha_{t}}$ \\
$X_{3}$ & 0 & 0 & 0 & 0 & 0 & 0 & $\frac{a}{b} X_{\alpha}$ \\
$X_{4}$ & $X_{1}$ & $2 X_{2}$ & 0 & 0 & $-X_{5}$ & $-2 X_{6}$ & $-X_{\alpha^{\prime}}$ \\
$X_{5}$ & $-X_{3}$ & $2 a X_{1}$ & 0 & $X_{5}$ & 0 & 0 & $-X_{\alpha^{\prime \prime}}$ \\
$X_{6}$ & $2 X_{5}$ & $-2 b X_{3}+4 a X_{4}$ & 0 & $2 X_{6}$ & 0 & 0 & $-X_{\alpha^{\prime \prime \prime}}$ \\
$X_{\alpha}$ & $X_{\alpha_{x}}$ & $X_{\alpha t}$ & $-\frac{a}{b} X_{\alpha}$ & $X_{\alpha^{\prime}}$ & $X_{\alpha^{\prime \prime}}$ & $X_{\alpha^{\prime \prime \prime}}$ & 0 \\
where $\alpha^{\prime}=x \alpha_{x}+2 t \alpha_{t}$, & $\alpha^{\prime \prime}=2 a t \alpha_{x}+\frac{a}{b} x \alpha$, & $\alpha^{\prime \prime \prime}=4 a t x \alpha_{x}+4 a t^{2} \alpha_{t}+\frac{a}{b}\left(x^{2}+2 b t\right) \alpha$.
\end{tabular}

The Lie group $G_{i}$ generated by $X_{i}$ can take advantage of the previously obtained transformation relationship $e^{\epsilon X_{i}}(x, t, u)=(\hat{x}, \hat{t}, \hat{u})$, such as $\hat{x}$ for $G_{1}$

$$
\hat{x}=e^{\epsilon X_{1}} x=e^{\epsilon \frac{\partial}{\partial x}} x=\left[1+\epsilon \frac{\partial}{\partial x}+\frac{1}{2} \epsilon^{2}\left(\frac{\partial}{\partial x}\right)^{2} . \cdots\right] x=x+\epsilon \frac{\partial x}{\partial x}=x+\epsilon .
$$


and $G_{3}$

$$
\hat{u}=e^{\epsilon X_{3}} u=e^{\epsilon \frac{\partial}{\partial u}} u=\left[1+\epsilon \frac{\partial}{\partial u}+\frac{1}{2} \epsilon^{2}\left(\frac{\partial}{\partial u}\right)^{2} . \cdots\right] u=u+\epsilon \frac{\partial u}{\partial u}=u+\epsilon .
$$

Other situations can be calculated the same. All Lie groups are listed below

$$
\begin{aligned}
G_{1}:(x, t, u) & \mapsto(x+\epsilon, t, u), \\
G_{2}:(x, t, u) & \mapsto(x, t+\epsilon, u), \\
G_{3}:(x, t, u) & \mapsto(x, t, u+\epsilon), \\
G_{4}:(x, t, u) & \mapsto\left(e^{\epsilon} x, e^{2 \epsilon} t, u\right), \\
G_{5}:(x, t, u) & \mapsto\left(x+2 \epsilon a t, t, u-\epsilon x-\epsilon^{2} t\right), \\
G_{6}:(x, t, u) & \mapsto\left(\frac{x}{1-4 \epsilon a t}, \frac{t}{1-4 \epsilon a t}, u-\frac{\epsilon x^{2}}{1-4 \epsilon a t}+\frac{b}{a} \ln \sqrt{1-4 \epsilon a t}\right), \\
G_{\alpha}:(x, t, u) & \mapsto\left(x, t, \frac{b}{a} \ln \left(e^{\frac{a u}{b}}+\epsilon \frac{a}{b} \alpha\right) .\right.
\end{aligned}
$$

It can be seen from the observation that the $G_{1}$ group is spatial translation, $G_{2}$ is time translation, $G_{3}$ is the translation of the dependent variable, $G_{4}$ is the extension transformation, $G_{5}$ is the Galileo (speed) transformation, $G_{6}$ is a true local transform group whose physical meaning is ambiguous but very important. It can be used to derive similarity solutions.

\section{EXACT GENERAL SOLUTIONS OF $u_{t}=a\left(u_{x}\right)^{2}+b u_{x x}$}

With Lie symmetry we can construct exact solutions. Since $G_{i}$ is a symmetric group, if $u=f(x, t)$ is a solution of the equation (1), then the solution $u^{(i)}$ corresponding to $G_{i}$ can be expressed. Reach

$$
\begin{aligned}
u^{(1)} & =f(x-\epsilon, t), \\
u^{(2)} & =f(x, t-\epsilon), \\
u^{(3)} & =f(x, t)+\epsilon, \\
u^{(4)} & =f\left(e^{-\epsilon} x, e^{-2 \epsilon} t\right) \\
u^{(5)} & =f(x-2 \epsilon a t, t)-\epsilon x+\epsilon^{2} a t \\
u^{(6)} & =f\left(\frac{x}{1+4 \epsilon t}, \frac{t}{1+4 \epsilon a t}\right)-\frac{\epsilon x^{2}}{1+4 \epsilon a t}-\frac{b}{a} \ln \sqrt{1+4 \epsilon a t}, \\
u^{(\alpha)} & =\frac{b}{a} \ln \left(e^{\frac{a f(x, t)}{b}}+\epsilon \frac{a}{b} \alpha(x, t)\right) .
\end{aligned}
$$

The most common symmetry group case is the linear combination $X=c_{1} X_{1}+\ldots+c_{6} X_{6}+X_{\alpha}$. The explicit representation of this group is very complicated. We can construct an exponential map $\mathfrak{g}: \mathfrak{g} \mapsto G$, ie

$$
\mathfrak{g}=\exp \left(X_{\alpha}\right) \prod_{i=1}^{6} \exp \left(\epsilon_{i} X_{i}\right)=\exp \left(X_{\alpha}\right) \cdot \exp \left(\epsilon_{1} X_{1}\right) \cdot \ldots \cdot \exp \left(\epsilon_{6} X_{6}\right)
$$

If $u=f(x, t)$ is known to be a solution to the equation (1), under the $\mathfrak{g}$ group transformation, the most general solution can be obtained as follows

$$
\begin{aligned}
u & =f\left(e^{-\epsilon_{4}} \frac{x-2 \epsilon_{5} a t}{1+4 \epsilon_{6} a t}-\epsilon_{1}, \frac{t \epsilon^{-2 \epsilon_{4}}}{1+4 \epsilon_{6} a t}-\epsilon_{2}\right) \epsilon_{3}-\epsilon_{5} \frac{x-\epsilon_{5} a t}{1+4 \epsilon_{6} a t}-\frac{\epsilon_{6} x^{2}}{1+4 \epsilon_{6} a t} \\
& -\frac{b}{a} \ln \sqrt{1+4 \epsilon_{6} a t}+\frac{b}{a} \ln \left[e^{\frac{a}{b} f(x, t)}+\epsilon_{6} \frac{a}{b} \alpha(x, t)\right] .
\end{aligned}
$$

This general solution is obtained by this paper and has never been apparent in the literature. 
IV. EXACT SIMILARITY SOLUTIONS OF $u_{t}=a\left(u_{x}\right)^{2}+b u_{x x}$

With an infinitesimal generator $X_{i}$, you can determine the corresponding invariant, and you can use the invariant to simplify the equation (1) to find its solution.

Notice that the combination of $X_{1}$ and $X_{2}$ produces a moving wave solution. Let the new variable $\mu=x-c t$, so $u=u(x, t)=f(\mu)$, where $c$ is the wave speed. Thus the equation (1) becomes

$$
b f^{\prime \prime}+a f^{\prime 2}+c f^{\prime}=0 .
$$

Where $f^{\prime}=\frac{d f}{d \mu}$. The solution of this equation can be integrated

$$
u(x, t)=\frac{b}{a} \ln \left[c_{1} e^{\frac{c}{b}(x-c t)}-\frac{a}{c}\right]-\frac{c}{a}(x-c t)+c_{2} .
$$

In the formula, $c_{1}, c_{2}$ are integral constants.

Corresponding to $G_{3}$, obviously, if $u=f(x, t)$ is a solution of the equation $u_{t}=a\left(u_{x}\right)^{2}+b u_{x x}$, then $u=f(x, t)+c$ is also the equation.

For the stretch group of $G_{4}$, you can derive invariants and similar variables

$$
\theta=x t^{-1 / 2}, \quad \omega=u \text {. }
$$

The Lie group invariant solution is $\omega=f(\theta)=f\left(x t^{-1 / 2}\right)$, ie

$$
u=f\left(x t^{-1 / 2}\right)
$$

Substituting this formula into (1) can be simplified into ordinary differential equations.

$$
b f^{\prime \prime}+a f^{\prime 2}+\frac{1}{2} \theta f^{\prime}=0
$$

where $f^{\prime}=\frac{d f}{d \theta}$ and

$$
f(\theta)=\int \frac{\exp \left(\frac{-\theta^{2}}{4 b}\right)}{\int \exp \left(\frac{-\theta^{2}}{4 b}\right) d \theta+p_{1}} d \theta+p_{2} .
$$

Liu at el. [23] did not complete the above integration and left as it is.

If you check the integration carefully, it is not hard to recognise that $\int \exp \left(\frac{-\theta^{2}}{4 b}\right) d \theta$ should be linkage with error function. After simple cancellation, the solution of the equation (43) can be expressed by error function [25].

$$
f(\theta)=\frac{b}{a} \ln \left\{\frac{a}{b}\left[c_{1} \sqrt{\pi} \sqrt{b} \cdot \operatorname{erf}\left(\frac{1}{2} \frac{\theta}{\sqrt{b}}\right)+c_{2}\right]\right\} .
$$

where $\operatorname{erf}(\theta)=\frac{2}{\pi} \int_{0}^{\theta} e^{-\theta^{2}} d \theta$ is error function.

For the Galileo group $G_{5}$, we have similar variables

$$
\theta=t, \quad \omega=\frac{1}{2} x^{2}+2 a t u
$$

This group invariant solution is $\omega=f(\theta)$, ie

$$
u=\frac{1}{2 a t} f(t)-\frac{1}{4 a t} x^{2} .
$$

Substituting (47) into (1) to obtain ordinary differential equations

$$
t f^{\prime}-f+b t=0
$$

where $f^{\prime}=\frac{d f}{d \theta}$ 。 By integration, we obtain its solution as follows

$$
u(x, t)=\frac{c_{1}}{2 a}-\frac{b}{2 a} \ln t-\frac{1}{4 a t} x^{2} .
$$


For the group $G_{6}$, the first set of similar variables can be obtained.

$$
\theta=x t^{-1}, \quad \omega=u+\frac{x^{2}}{4 a t}+\frac{b}{2 a} \ln t .
$$

This Lie group invariant solution is

$$
u=f\left(x t^{-1}\right)-\frac{x^{2}}{4 a t}-\frac{b}{2 a} \ln t .
$$

Substituting (51) into (1) gives an ordinary differential equation

$$
b f^{\prime \prime}+a f^{\prime 2}=0
$$

by integration

$$
\omega=\frac{b}{a} \ln \left|\frac{a}{b} \theta+c_{1}\right|+c_{2} .
$$

So, in this case corresponding to $G_{6}$, there is a solution.

$$
u(x, t)=\frac{b}{a} \ln \left|\frac{a}{b} x t^{-1}+c_{1}\right|-\frac{x^{2}}{4 a t}-\frac{b}{2 a} \ln t+c_{2} .
$$

Corresponding to $G_{6}$, another similar variable, the $\ln t$ in the expression (50) is changed to $\ln x$, and the second set of similar variables is

$$
\theta=x t^{-1}, \quad \omega=u+\frac{x^{2}}{4 a t}+\frac{b}{2 a} \ln x .
$$

At this time, the Lie group invariant solution is

$$
u=f\left(x t^{-1}\right)-\frac{x^{2}}{4 a t}-\frac{b}{2 a} \ln x
$$

Substituting the expression (56) into the original equation (1) yields the equation for the function $f(\theta)$

$$
4 a b \theta^{2} f^{\prime \prime}+4 a^{2} \theta^{2} f^{\prime 2}-4 a b \theta f^{\prime}+3 b^{2}=0 .
$$

where $f^{\prime}=\frac{d f}{d \theta}$. Liu at el. [23] pointed out that the exact and explicit solutions of equation (57) cannot be obtained and be expressed by elementary functions, so they propose a creative series solution.

In fact, this equation can be solved and has the following exact and explicit elementary solution:

$$
f(\theta)=\frac{b}{2 a} \ln \left[\left(\frac{a}{b}\right)^{2} \theta\left(c_{1} \theta-c_{2}\right)^{2}\right]
$$

So there is a second form of exact solution

$$
u(x, t)=\frac{b}{2 a} \ln \left[\left(\frac{a}{b}\right)^{2} \frac{x}{t}\left(c_{1} \frac{x}{t}-c_{2}\right)^{2}\right]-\frac{x^{2}}{4 a t}-\frac{b}{2 a} \ln x .
$$

The exact solution of the equations (58) and (59) are obtained by this paper [25].

\section{NUMERICAL CALCULATIONS OF $u_{t}=\left(u_{x}\right)^{2}+u_{x x}$ AND DISCUSSIONS}

For equation $u_{t}=\left(u_{x}\right)^{2}+u_{x x}$, its solution is $u(x, t)=\frac{1}{2} \ln \left[\frac{x}{t}\left(c_{1} \frac{x}{t}-c_{2}\right)^{2}\right]-\frac{x^{2}}{4 t}-\frac{1}{2} \ln x$. As an example, we have initial conditions: $u(1,1)=1$ and $u(1,2)=1$. Hence, $c_{1}=e^{2}-e^{3 / 2}$ and $c_{2}=e^{2}-2 e^{5 / 4}$. The solution is plotted in Figure 1 and 1 . It is worth mentioning that the above solutions have never been apparent in the literature.

The numerical simulations indicate that the solution is in a rapid oscillation and tends to zero in a long-time.

In summary, the three new solutions have been obtained in this paper are listed in the Table below.

\section{ACKNOWLEDGEMENT}

It is my great pleasure to have shared and discussed some thoughts of this paper with Michael Sun from Bishops Diocesan College, whose pure and direct scientific sense inspired me. This paper is dedicated to the memory my beloved father, Zhong-Chuan Sun. 


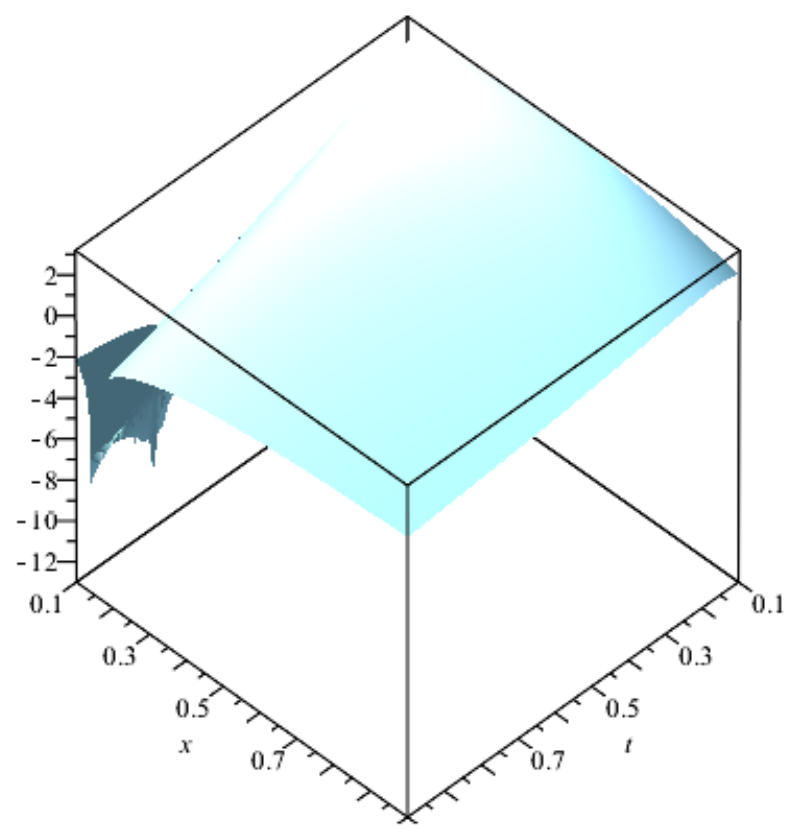

FIG. 1: The solution is quite oscillation near the origin $(0,0)$.

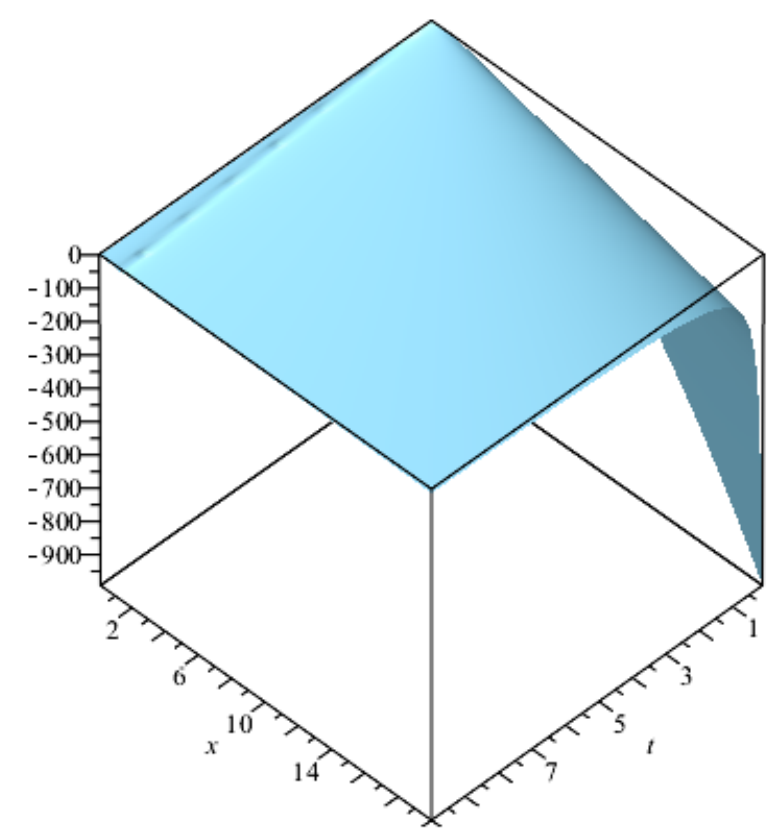

FIG. 2: The solution tends to zero when it is far from the origin $(0,0)$.

TABLE II: Three new solutions obtained in this paper \begin{tabular}{c|c}
\hline General solution & Eq.(38) \\
\hline
\end{tabular}

\begin{tabular}{c|c}
\hline Group G4 & Eq.(45) \\
\hline Group G6 & Eq.(59) \\
\hline
\end{tabular}


[1] S. Lie, Theorie der Transformationsgruppen I (in German). Leipzig: B.G.Teubner. Written with the help of Friedrich Engel. 1888.

[2] S. Lie, Theorie der Transformationsgruppen II(in German), Leipzig: B.G.Teubner. Written with the help of Friedrich Engel. 1890.

[3] S. Lie, Theorie der Transformationsgruppen III (in German), Leipzig: B. G. Teubner. Written with the help of Friedrich Engel. 1893.

[4] B.J. Cantwell, Introduction to Symmetry Analysis, Cambridge:Cambridge University Press, 2002.

[5] J.M. Page, Ordinary differential equation, with an introduction of Lie theory of the group of one parameter. Macmillan and Co.Limited, 1897.

[6] A. Cohen, An Introduction to the Lie Theory of one-point parameter groups, D.C.: Heath and Co. Publishers,1911.

[7] E.I. Ince, Ordinary Differential Equations, New York:Dover Publications, 1926.

[8] G. Birkhoff, Hydrodynamics, Princeton:Princeton University Press, 1950.

[9] L.V. Ovsiannikov, Group Analysis of Differential Equations, Moscow:Nauka, 1962. English translation by Ames, W.F. (ed.), New York:Academic Press, 1982.

[10] W.F. Ames, Nonlinear Partial Differential Equations in Engineering, New Yoyk:Academic Press,1965.

[11] P.J. Olver, Applications of Lie Groups to Differential Equations, New York:Springer,1986.

[12] N.K. Ibragimov, Group analysis of ordinary differential equations and the invariance principle in mathematical physics, Russian Math. Surveys. 1992, 47(2):89-156.

[13] N.H. Ibragimov, Mahomed F.M. CRC Handbook of Lie Group Analysis of Differential Equations, vol. 3, Boca Raton:CRC Press, 1996.

[14] N.H. Ibragimov, A new conservation theorem, J. Math. Anal. Appl. 2007,333:311-328.

[15] R.L. Anderson and N.H. Ibragimov, Lie-Bäcklund Transformations in Applications, Philadephia:SIAM, 1979.

[16] G.W. Bluman and S. Anco, Symmetry and Integration Methods for Differential Equations, New York:Springer-Verlag,2002.

[17] J.M. Hill, Differential equations and group methods for scientists and engineers, CRC Press, 1992.

[18] T. Hawkins, Emergence of the theory of Lie groups, New York: Springer-Verlag, 2000.

[19] P. Hydon, Symmetry Methods for Differential Equations: A Beginners Guide, Cambridge University Press, 2000.

[20] G.Emanuel, Solution of Ordinary Differetial Equations by Continuous Groups, Florida: Chapman \& Hall/CRC, 2001.

[21] H. Stephani, Differential Equations-Their Solution Using Symmetries, New York:Cambridge University Press, 1989.

[22] J. M. Burgers. A mathematical model illustrating the theory of turbulence. Advances in Applied Mechanics, Vol. I, 171-199, Academic, New York, 1948.

[23] H.Z. Liu, J.B. Li and Q.X. Zhang, Lie symmetry analysis and exaxt explicit solutions for general Burgers equation. J. of Compoutational and Applied Mathematics. 228:1-9. 2009.

[24] Z.J Cao, Y.P. Lin, Lie point symmetries, conservation laws, and solutions of a space dependent reaction - diffusion equation. Applied Mathematics and Computation. 2012, 248:386 - 398.

[25] B. Sun, Exact solution of general Burgers equation for the genuinely local group of transformation. DOI: 10.13140/RG.2.1.1043.5923. (2015-12-1) https://www.researchgate.net/publication/285322511.

[26] B. Sun, Noether conservation laws in elasticity, DOI: 10.13140/RG.2.1.1243.6560 (2016-1-13) https://www.researchgate.net/publication/290306379

[27] B. Sun, Dimensional Alysis and Lie Group, China Higher Educational Press, Beijing, 2016. 